\title{
Dextromethorphan/Bupropion: A Novel Oral NMDA (N-methyl-d-aspartate) Receptor Antagonist with Multimodal Activity
}

\author{
Stephen M. Stahl
} ISSUE:

Although currently available antidepressants increase monoamine levels soon after the start of treatment, therapeutic benefits are often delayed by several weeks and the majority of patients with major depressive disorder fail to achieve an adequate response to first- or second-line therapies targeting monoamines. The recent approval of the NMDA (N-methyl-d-aspartate) antagonist esketamine given intranasally for treatment-resistant depression has reinforced the need for agents with rapid onset with alternate mechanisms of action. Dextromethorphan/bupropion, an investigational medicine currently in development, is one such candidate.

\section{Take-Home Points}

- The therapeutic actions of traditional antidepressants are often delayed by weeks and are associated with significant side effects.

- The use of agents with more rapid onsets of action, such as ketamine, is restricted due to the need for monitoring, risk of serious side effects, and the potential for abuse/misuse.

- Dextromethorphan has similar pharmacological properties to ketamine and is primarily thought to act through a combination of activities at the N-methyl-Daspartate (NMDA) receptor, serotonin transporter (SERT), norepinephrine transporter (NET), and sigma-1 receptor. When combined with bupropion, a wellestablished antidepressant, there may be a synergistic therapeutic effect. Bupropion primarily acts through NET and dopamine transporter (DAT) with additional cytochrome P450 2D6 (CYP450 2D6) inhibition.

- In contrast to ketamine, dextromethorphan/bupropion is orally administered and does not appear to induce dissociative effects at therapeutically active concentrations.

\section{Introduction}

A novel, oral, investigational NMDA (N-methyl-d-aspartate) receptor antagonist with multimodal activity is currently being developed for the treatment of central nervous system disorders. This agent combines the NMDA antagonist dextromethorphan with the norepinephrine-dopamine reuptake inhibitor bupropion. ${ }^{1}$ Dextromethorphan is rapidly metabolized by cytochrome P450 2D6 (CYP2D6), making it difficult to achieve therapeutic plasma levels following oral administration. ${ }^{2}$ Bupropion and its metabolites are CYP2D6 inhibitors ${ }^{3}$ and the administration of bupropion in combination with dextromethorphan leads to significant increases in dextromethorphan exposure at all evaluated doses in three Phase I studies (Figure 1). ${ }^{4}$ Bupropion, with its distinct centrally-acting mechanisms of action, is a compelling choice as metabolic inhibitor of dextromethorphan and suggests the potential for pharmacological synergy and clinical use across a broad range of neuropsychiatric conditions. Dextromethorphan/bupropion is currently in latestage clinical development for major depressive disorder (including treatment-resistant depression), Alzheimer's disease agitation, and smoking cessation. ${ }^{4}$ 
Figure 1. Dextromethorphan is rapidly metabolized by CYP2D6 following oral administration in humans. Co-administration with a CYP2D6 inhibitor, such as bupropion, inhibits dextromethorphan metabolism, maintaining dextromethorphan concentrations in the therapeutic range.

Dextromethorphan (CYP2D6 Substrate)

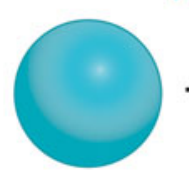

Rapid metabolism

Bupropion

(CYP2D6 Inhibitor)

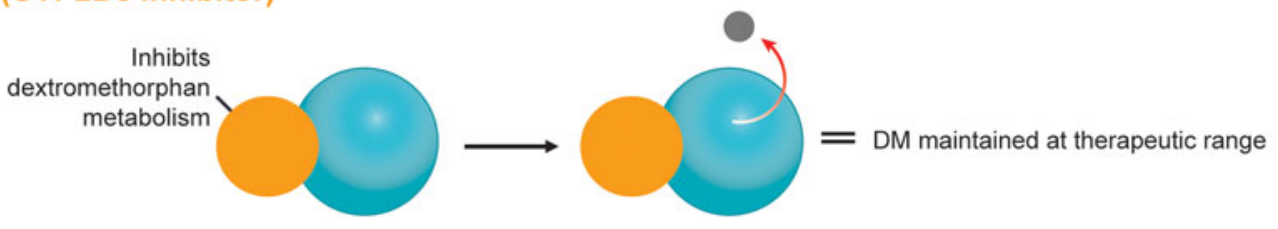

\section{Beyond Monoamines: Unconventional Antidepressant Mechanisms and Multimodal Therapeutic Approaches}

The classic monoamine hypothesis of depression posits in an oversimplified manner that depression arises due to a deficiency in monoamine neurotransmitters. Although direct evidence for the monoamine hypothesis is lacking, virtually all currently available antidepressants directly affect one or more monoamine neurotransmitter systems. However, despite the fact that such agents increase monoamine levels shortly after initiating treatment, the therapeutic benefits are often delayed by several weeks. ${ }^{1}$

As a result, the focus has now shifted away from the neurotransmitters themselves to their receptors and the downstream molecular events that these receptors trigger in order to explain the mechanism of action of antidepressants. For example, antidepressant-mediated alterations in neurogenesis and neuroplasticity have drawn increased attention. Treatment with agents targeting the monoamine pathway, such as selective serotonin reuptake inhibitors (SSRIs), has been shown to increase neurogenesis in the dentate gyrus of the hippocampus. ${ }^{5}$ These effects may be mediated by activating cAMP (cyclic adenosine monophosphate) response element binding protein (CREB), which can elicit the expression of numerous genes involved in neuroplasticity, including brain-derived neurotrophic factor (BDNF). ${ }^{1}$ Clinical studies have indicated that chronic antidepressant treatment can restore abnormally low BDNF levels and such restoration has been correlated with reduced scores on depression rating scales. ${ }^{6}$

In addition, glutamatergic signaling is thought to play a role in maintaining neuroplasticity. Glutamatemediated activation of AMPA ( $\alpha$-amino-3-hydroxy-5methyl-4-isoxazolepropionic acid) receptors leads to activation of the extracellular signal-regulated kinase/protein kinase B, or ERK/AKT signal transduction cascade, triggering the mammalian target of rapamycin (mTOR) pathway. Activation of the mTOR pathway has been reported to increase synaptic protein expression, resulting in increased dendritic spine density. ${ }^{1,7}$ Synaptic and intracellular events stimulated by ketamine suggest that NMDA antagonism produces a "glutamate surge" that is then hypothesized to increase AMPA/NMDA receptor flux, stimulating intracellular signaling cascades (Figure 2). Studies have shown that both ketamine and dextromethorphan require activation of AMPA receptors to mediate their antidepressant effects. $1,8,9,10,11$

Dextromethorphan/bupropion combines the mechanisms of action of several distinct antidepressant therapeutic classes into one therapeutic agent. Both dextromethorphan and bupropion increase norepinephrine availability by inhibiting reuptake and acting as alpha4-beta-2 nicotinic (nACh) antagonists. In addition, bupropion increases dopamine availability by blocking reuptake, while dextromethorphan increases glutamate by acting as an NMDA receptor antagonist and serotonin (by both acting as a serotonin reuptake inhibitor and boosting serotonin activity in the dorsal raphe via sigma-1 agonism) (Figure 3). ${ }^{1}$ 


\section{BRAINSTORMS-Clinical Neuroscience Update}

Figure 2. NMDA blockade-mediated activation of AMPA receptors induces downstream cascades involved in neural plasticity that are hypothesized to underlie antidepressant effects.

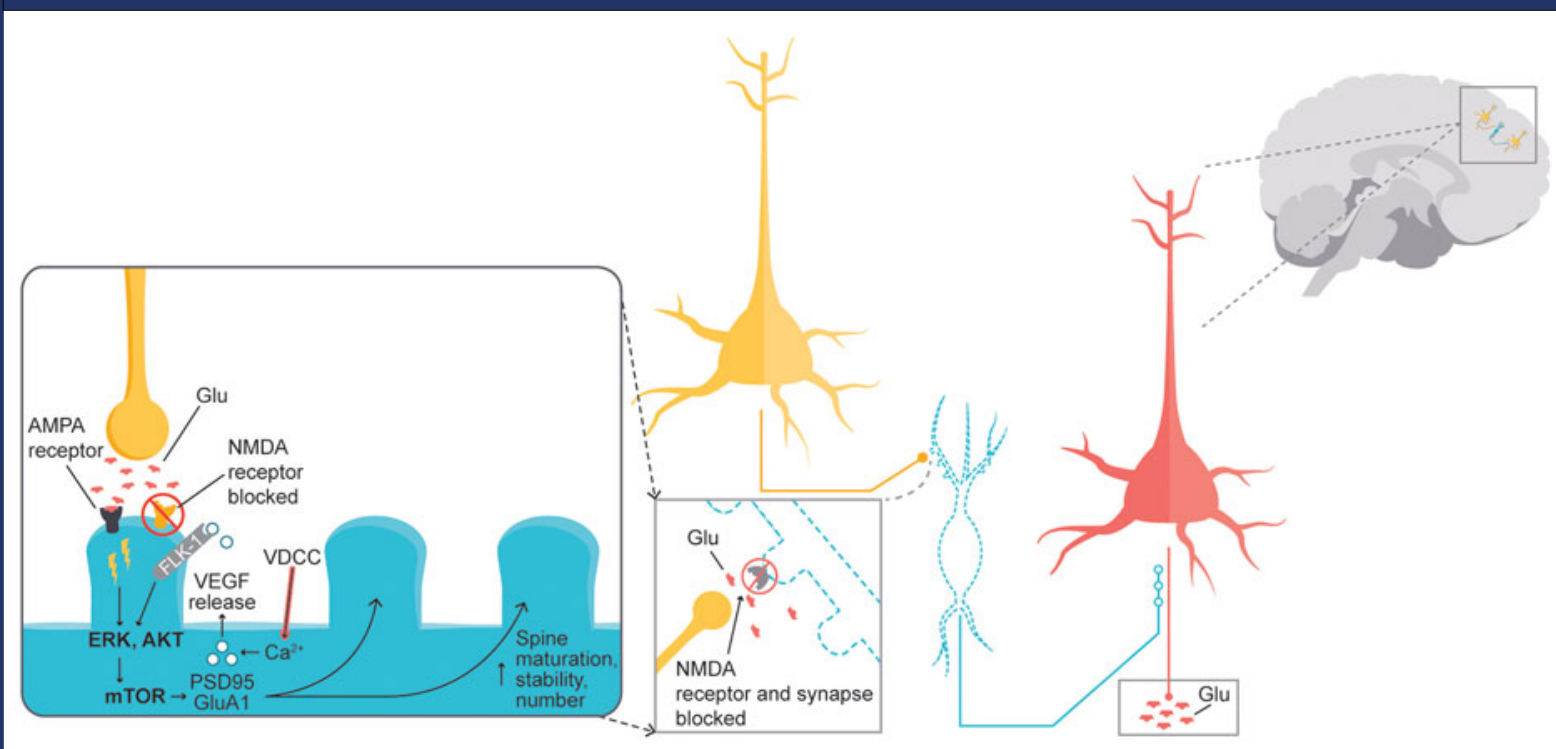

Glu = glutamate $:$ NMDA $=\mathrm{N}$-methyl-D-aspartate; $\mathrm{AMPA}=\mathrm{\alpha}$-amino-3-hydroxy-5-methyl-4-isoxazolepropionic acid; $\mathrm{PSD} 95=$ postsynaptic density protien 95 $\mathrm{VDCC}=$ voltage-dependent calcium channel; VEGF = vascular endothelial growth factor

Figure 3. Dextromethorphan (Panel A) is an NMDA receptor antagonist that acts as a SERT and NET inhibitor, nACh $\alpha 4 \beta 4$ antagonist, sigma-1 and mu opioid receptor agonist. Bupropion (Panel B) is a CYP2D6 inhibitor that acts as a NET and DAT inhibitor and a nACh $\alpha 4 \beta 4$ antagonist.

(a)

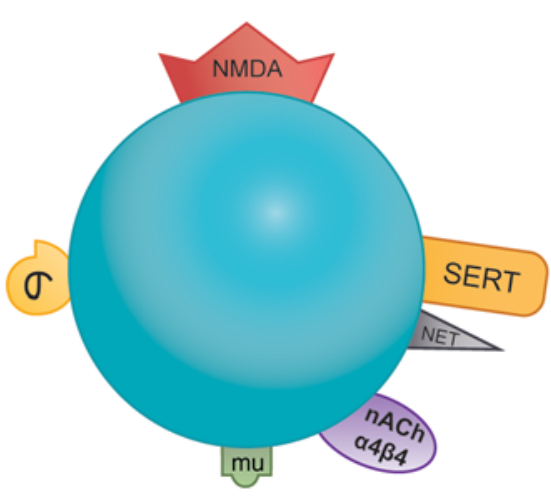

(b)

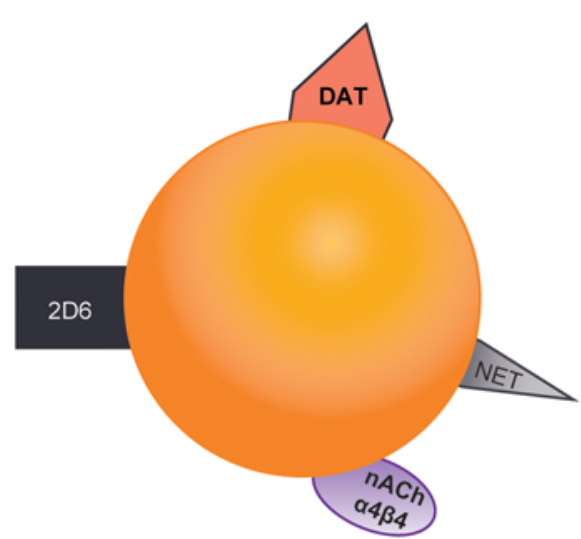

SERT = Serotonin Reuptake Transporter; NET = Norepinephrine Reuptake Transporter; nACh = Nicotinic Acetylcholine Receptor; CYP 450 2D6 = Cytochrome P450 2D6; NMDA = N-methyl-D-aspartate; DAT = Dopamine Reuptake Transporter; $\sigma=$ Sigma- 1 ; mu = mu Opioid Receptor 
Figure 4. The pharmacological properties of ketamine and dextromethorphan share significant overlap. Although both agents have affinity for the NMDA receptor, the sigma-1 potency and NMDA receptor affinity of dextromethorphan are higher than those of ketamine in some cellular assay systems.

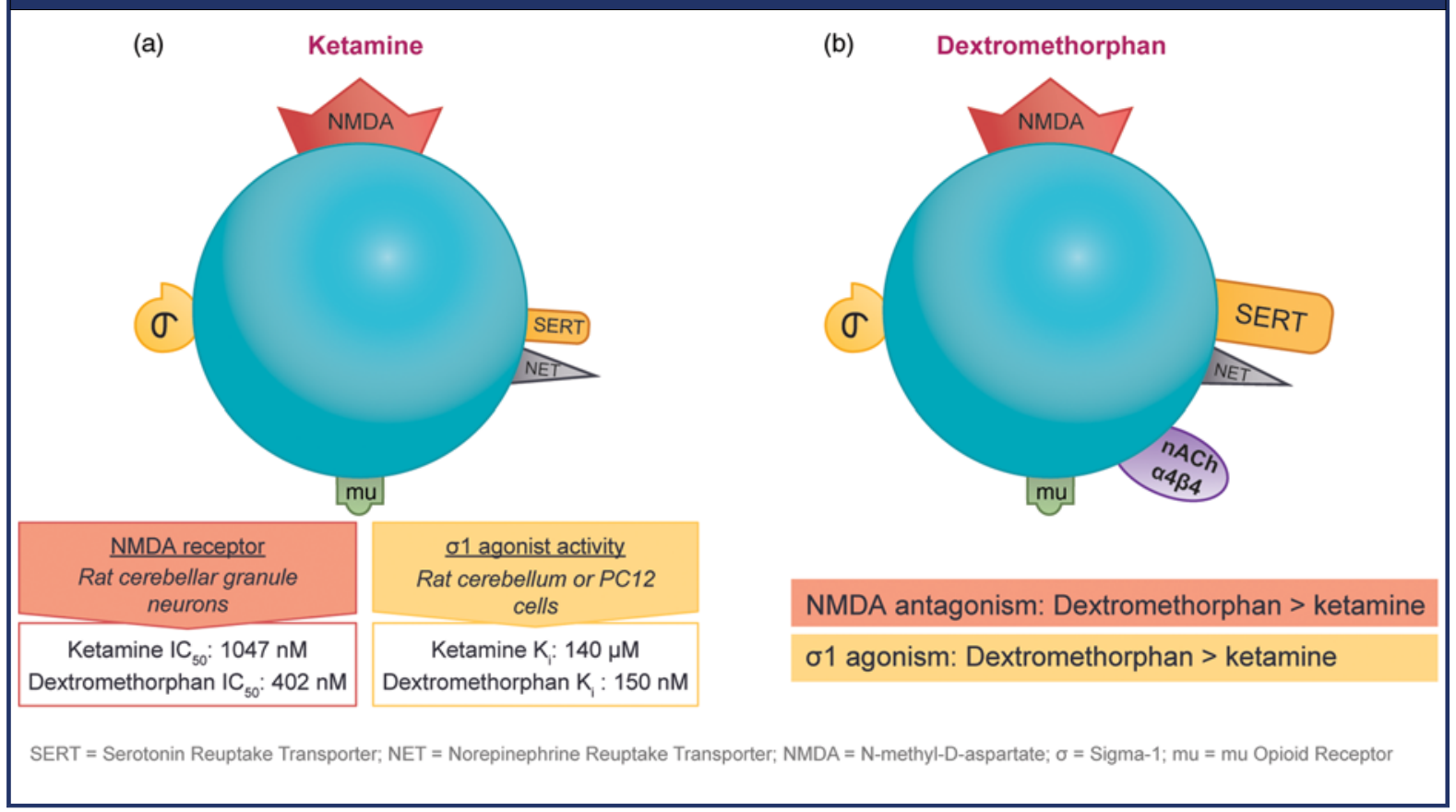

The observation that subanesthetic doses of ketamine induce immediate antidepressant effects in patients with treatment-resistant unipolar or bipolar depression has spurred the search for agents with similar pharmaceutical properties. Ketamine and dextromethorphan display activity at the same receptors; however, dextromethorphan exhibits both higher affinity as an NMDA receptor antagonist and greater potency at the sigma-1 site than ketamine in some cellular assay systems (Figure 4). 1,12,13,14,15

Interestingly, opioid receptor antagonism via administration of naltrexone prior to the intravenous administration of ketamine attenuated the antidepressant effects (but not the dissociative effects) of ketamine in adults with treatment-resistant depression, suggesting that the acute antidepressant effects of ketamine require opioid activation but the dissociative effects do not. ${ }^{16}$ One explanation for this observation is that crosstalk between opioid receptor-mediated signaling and NMDA receptor-mediated signaling interferes with ketamine's ability to activate mTOR. ${ }^{17}$ However, treatment of patients with the mTOR inhibitor rapamycin prior to ketamine infusion potentiated rather than blocked the antidepressant effects of ketamine. ${ }^{18}$ Therefore, further research is required to fully elucidate the role of opioid signaling in the antidepressant effects of ketamine. Based on several studies examining the effect of naloxone, an opioid receptor antagonist, mu opioid receptor activity has not been shown to be clinically relevant to dextromethorphan, however the antidepressant effect has not been examined. ${ }^{19,20}$

\section{Considerations for Major Depressive Disorder and Treatment-Resistant Depression}

Major depressive disorder continues to be a leading cause of disease burden, both in the United States and globally. In 2017, an estimated 17.3 million adults over the age of 18 in the United States experienced at least 1 major depressive episode, while over 300 million individuals worldwide are estimated to suffer from depression. ${ }^{21,22}$ Studies indicate that the majority of patients inadequately respond to monoamine-targeting therapy. Approximately $63 \%$ of patients failed to achieve remission after initial treatment with an SSRI. ${ }^{23}$ And of those patients who failed to remit, $69 \%$ failed to achieve remission upon switching to a second antidepressant. ${ }^{23}$ In patients who do achieve remission, residual symptoms, including insomnia, weight gain, and impaired concentration/decision making, are common. ${ }^{24,25}$ 
At present, only a few treatment options have been FDA-approved for treatment-resistant depression or as an adjunct to antidepressants for major depressive disorder: SYMBYAX $($ ) (olanzapine in combination with fluoxetine), SPRAVATO ${ }^{\mathrm{TM}}$ (esketamine), ABILIFY@ (aripiprazole), and REXULTI $($ brexpiprazole). ${ }^{26,27,28,29}$ However, the use of SPRAVATO ${ }^{\text {TM }}$ in patients with treatment-resistant depression has been restricted to approved healthcare settings due to the risks of sedation, dissociation, and abuse/misuse, highlighting the need for more easily accessible agents with fewer side effects. ${ }^{27}$

Research suggests that combination therapy with multi-modal agents is more effective than antidepressant monotherapy. While $25 \%$ of patients treated with an SSRI alone for 6 weeks achieved remission, $52 \%$ of those treated with an SSRI in combination with a noradrenergic and specific serotonergic antidepressant (NaSSA) and $46 \%$ of those treated with an NaSSA in combination with bupropion achieved remission. ${ }^{30}$

The multimodal pharmacological activity of dextromethorphan/bupropion therapy also contributes to more rapid efficacy than antidepressant monotherapy. In the Phase 2, multicenter, randomized, double-blind, active-controlled ASCEND (Assessing Clinical Episodes in Depression) trial, 80 patients with a confirmed diagnosis of moderate to severe major depressive disorder were randomized in a 1:1 ratio to receive $45 \mathrm{mg}$ dextromethorphan/105 mg bupropion $(\mathrm{n}=43)$ or bupropion $(105 \mathrm{mg})(\mathrm{n}=37)$ twice daily for 6 weeks. Treatment with dextromethorphan/bupropion resulted in significantly lower Montgomery-Åsberg Depression Rating Scale (MADRS) total scores by week 2 as compared to bupropion monotherapy. In addition, $26 \%$ of patients treated with dextromethorphan/bupropion had achieved remission by week 2 versus $3 \%$ of those receiving bupropion monotherapy. Notably, no NMDA dissociative/psychotomimetic events were observed in the ASCEND trial. $^{31}$

\section{Summary and Conclusions}

Dextromethorphan/bupropion is an orally administered, rapidly-acting, investigational NMDA receptor antagonist with multimodal activity. The formulation achieves pharmacologic synergy by simultaneously targeting monoamines, NMDA receptors, and sigma- 1 receptors, resulting in more rapid and robust decreases in depression rating scale scores than bupropion treatment alone. With its similar pharmacological properties to ketamine, dextromethorphan/bupropion represents a promising new investigational agent for depression.

\section{Disclosure information}

Stephen M. Stahl, M.D., PhD, Dsc (Hon.) is an Adjunct Professor of Psychiatry at the University of California San Diego, Honorary Visiting Senior Fellow at the University of Cambridge, UK and Director of Psychopharmacology for California Department of State Hospitals. Over the past 36 months (January 2016 - December 2018) Dr. Stahl has served as a consultant to Acadia, Adamas, Alkermes, Allergan, Arbor Pharmaceutcials, AstraZeneca, Avanir, Axovant, Axsome, Biogen, Biomarin, Biopharma, Celgene, Concert, ClearView, DepoMed, Dey, EnVivo, EMD Serono, Ferring, Forest, Forum, Genomind, Innovative Science Solutions, Intra-Cellular Therapies, Janssen, Jazz, Lilly, Lundbeck, Merck, Neos, Novartis, Noveida, Orexigen, Otsuka, PamLabs, Perrigo, Pfizer, Pierre Fabre, Reviva, Servier, Shire, Sprout, Sunovion, Taisho, Takeda, Taliaz, Teva, Tonix, Trius, Vanda, Vertex and Viforpharma; he has been a board member of RCT Logic and Genomind; he has served on speakers bureaus for Acadia, Astra Zeneca, Dey Pharma, EnVivo, Eli Lilly, Forum, Genentech, Janssen, Lundbeck, Merck, Otsuka, PamLabs, Pfizer Israel, Servier, Sunovion and Takeda and he has received research and/or grant support from Acadia, Alkermes, AssureX, Astra Zeneca, Arbor Pharmaceuticals, Avanir, Axovant, Biogen, Braeburn Pharmaceuticals, BristolMyer Squibb, Celgene, CeNeRx, Cephalon, Dey, Eli Lilly, EnVivo, Forest, Forum, GenOmind, Glaxo Smith Kline, Intra-Cellular Therapies, ISSWSH, Janssen, JayMac, Jazz, Lundbeck, Merck, Mylan, Neurocrine, Neuronetics, Novartis, Otsuka, PamLabs, Pfizer, Reviva, Roche, Sepracor, Servier, Shire, Sprout, Sunovion, TMS NeuroHealth Centers, Takeda, Teva, Tonix, Vanda, Valeant and Wyeth.

\section{References:}

1 Stahl SM. Stahl's Essential Psychopharmacology: Neuroscientific Basis and Practical Applications. 4th ed. New York, NY: Cambridge University Press; 2013.

2 NUEDEXTA® Prescribing Information. Aliso Viejo, CA: Avanir Pharmaceuticals, Inc.; January 2019.

3 WELLBUTRIN@ Prescribing Information. Research Triangle Park, NC: GlaxoSmithKline; August 2017

4 O'Gorman C, Iosifescu DV, Jones A, et al. Clinical development of AXS-05 for treatment resistant depression and agitation associated with Alzheimer's disease. P7-122. Presented at the APA Annual Meeting 2018; May 5-9, 2018; New York, NY.

5 Crupi R, Marino A, and Cuzzocrea S. New Therapeutic Strategy for Mood Disorders. Curr Med Chem. 2011; 18(28):4284-98.

6 Sen S, Duman R, and Sanacora G. Serum brain-derived neurotrophic factor, depression, and antidepressant medications: Meta-analyses and implications. Biol Psychiatry 2008; 64:527-532. 


\section{BRAINSTORMS-Clinical Neuroscience Update}

7 Deyama S, Bang E, Wohleb ES, et al. Role of neuronal VEGF signaling in the prefrontal cortex in the rapid antidepressant effects of ketamine. Am J Psychiatry. 2019;176:388-400.

8 Niciu MJ, Ionescu DF, Richards EM, Zarate CA. Glutamate and its receptors in the pathophysiology and treatment of major depressive disorder. J Neural Transm (Vienna). 2014;121(8):907-924.

9 Freudenberg F, Celikel T, Reif A. The role of $\alpha$-amino-3hydroxy-5-methyl-4-isoxazolepropionic acid (AMPA) receptors in depression: central mediators of pathophysiology and antidepressant activity? Neurosci Biobehav Rev. 2015;52:193-206.

10 Zarate C, Machado-Vieira R, Henter I, et al. Glutamatergic modulators: the future of treating mood disorders? Harv Rev Psychiatry. 2010;18(5):293-303.

11 Nguyen L, Matsumoto RR. Involvement of AMPA receptors in the antidepressant-like effects of dextromethorphan in mice. Behav Brain Res. 2015;295:26-34.

12 Berman FW, Murray TF. Characterization of [3H]MK-801 binding to N-methyl-D-aspartate receptors in cultured rat cerebellar granule neurons and involvement in glutamatemediated toxicity. J Biochem Toxicol. 1996;11(5):217-226.

13 Werling LL, Keller A, Frank JG, Nuwayhid SJ. A comparison of the binding profiles of dextromethorphan, memantine, fluoxetine and amitriptyline: treatment of involuntary emotional expression disorder. Exp Neurol. 2007;207(2):248-257.

14 Robson MJ, Elliott M, Seminerio MJ, Matsumoto RR. Evaluation of sigma $(\sigma)$ receptors in the antidepressant-like effects of ketamine in vitro and in vivo. Eur Neuropsychopharmacol. 2012;22(4):308-317.

15 Lauterbach EC. An extension of hypotheses regarding rapidacting, treatment-refractory, and conventional antidepressant activity of dextromethorphan and dextrorphan. Med Hypotheses. 2012;78(6):693-702.

16 Williams NR, Heiferts BD, Blasey C, et al. Attenuation of antidepressant effects of ketamine by opioid receptor antagonism. Am J Psychiatry. 2018;175(12):1205-1215.

17 Wang M and Kaplin A. Explaining naltrexone's interference with ketamine's antidepressant effect. Am J Psychiatry. 2019;176(5):410-11.

18 Heiferts BD, Williams NR, Blasey C, et al. Rigorous translational models are key to studying ketamine's antidepressant mechanism: Response to Wang and Kaplin. Am J Psychiatry. 2019;176(5):412.
19 Gaginella TS, Bertko RJ, Kachur JF. Effect of dextromethorphan and levomethorphan on gastric emptying and intestinal transit in the rat. J Pharmacol Exp Ther. 1987;240(2):388-391.

20 Kachur JF, Morgan DW, Gaginella TS. Effect of dextromethorphan on guinea pig ileal contractility in vitro: comparison with levomethorphan, loperamide, and codeine. J Pharmacol Exp Ther. 1986;239(3):661-667.

21 National Institute of Mental Health. Major depression. NIMH website. https://www.nimh.nih.gov/health/statistics/majordepression.shtml. Updated February 2019. Accessed May 19, 2019.

22 World Health Organization. Fact sheets: depression. WHO website. https:/ /www.who.int/news-room/fact-sheets/detail/ depression Published March 22, 2018. Accessed May 19, 2019.

23 Rush AJ, Trivedi MH, Wisniewski SR, et al. Acute and longerterm outcomes in depressed outpatients requiring one or several treatment steps: a STAR*D report. Am J Psychiatry. 2006;163(11):1905-1917.

24 Nierenberg AA, Husain MM, Trivedi MH, et al. Residual symptoms after remission of major depressive disorder with citalopram and risk of relapse: a STAR*D report. Psychol Med. 2010;40(1):41-50.

25 McClintock SM, Husain MM, Wisniewski SR, et al. Residual symptoms in depressed outpatients who respond by $50 \%$ but do not remit to antidepressant medication. J Clin Psychopharmacol. 2011;31(2):180-186.

26 SYMBYAX@ Prescribing Information. Indianapolis, IN: Lilly USA, LLC; March 2018.

27 SPRAVATO ${ }^{\text {TM }}$ Prescribing information. Titusville, NJ: Janssen Pharmaceuticals; May 2019.

28 ABILIFY@ Prescribing Information. Tokyo, Japan: Otsuka Pharmaceutical Co., Ltd; February 2018.

29 REXULTI@ Prescribing Information. Tokyo, Japan: Otsuka Pharmaceutical Co., Ltd; February 2018.

30 Blier P, Ward HE, Tremblay P, et al. Combination of antidepressant medications from treatment initiation for major depressive disorder: a double-blind randomized study. $A m \mathrm{~J}$ Psychiatry. 2010;167(3):281-288

31 Anderson A, Iosifescu DV, Jacobson M, et al. Efficacy and safety of AXS-05, an oral NMDA receptor antagonist with multimodal activity, in major depressive disorder: results of a phase 2, double-blind, active-controlled trial. W43. Presented at the ASCP Annual Meeting 2019; May 28-31, 2019; Scottsdale, AZ. 